\title{
The Exponential Flexible Weibull Extension Distribution
}

\author{
Beih S. El-Desouky, Abdelfattah Mustafa, Shamsan Al-Garash \\ Department of Mathematics, Faculty of Science, Mansoura University, Mansoura, Egypt \\ Email: b_desouky@yahoo.com, abdelfatah_mustafa@yahoo.com, shamsan_algarash@hotmail.com
}

How to cite this paper: El-Desouky, B.S., Mustafa, A. and Al-Garash, S. (2017) The Exponential Flexible Weibull Extension Distribution. Open Journal of Modelling and Simulation, 5, 83-97.

http://dx.doi.org/10.4236/ojmsi.2017.51007

Received: November 2, 2016

Accepted: January 9, 2017

Published: January 12, 2017

Copyright (c) 2017 by authors and Scientific Research Publishing Inc. This work is licensed under the Creative Commons Attribution International License (CC BY 4.0).

http://creativecommons.org/licenses/by/4.0/

\begin{abstract}
This paper is devoted to study a new generalization of the flexible Weibull with three parameters. This model is referred to as the exponential flexible Weibull extension (EFWE) distribution which exhibits bathtub-shaped hazard rate function. Some statistical properties such as the mode, median, the $r$ th moment, quantile function, the moment generating function and order statistics are discussed. Moreover, the maximum likelihood method for estimating the model parameters and the Fisher's information matrix is given. Finally, the advantage of the EFWE distribution is concluded by an application using real data.
\end{abstract}

\section{Keywords}

Exponential Flexible Weibull, Exponential Distribution, Flexible Weibull Distribution, Exponential Weibull, Reliability, Moments, Estimation

\section{Introduction}

The Weibull distribution (WD) submitted by Weibull [1], is an important and popular distribution for modeling lifetime data where the hazard rate function is monotone. Recently, new classes of distributions were based on modifications of the Weibull distribution (WD) to provide a good fit to data set with bathtub hazard failure rate Xie and Lai [2]. The Modified Weibull (MW) distribution has been derived by Lai et al. [3] and Sarhan and Zaindin [4]. Moreover the Beta-Weibull (BW) distribution has been studied by Famoye et al. [5], Beta modified Weibull (BMW) distribution, see Silva et al. [6] and Nadarajah et al. [7] and Kumaraswamy Weibull (KW) distribution, see Cordeiro et al. [8]. Furthermore, many generalizations of the Weibull distribution are investigated like a Generalized modified Weibull (GMW) distribution, Carrasco et al. [9] and Exponentiated modified Weibull extension (EMWE) distribution, Sarhan and Apaloo [10]. Also we can find good review of these models in Pham and Lai [11] and Murthy et al. [12]. 
The Flexible Weibull (FWE) distribution (Bebbington et al. [13]) has many applications in life testing experiments, applied statistics, reliability analysis and clinical studies. For more details on this distribution, see [13]. If $X$ is a random variable and it has the Flexible Weibull Extension (FWE) distribution with parameters $\alpha, \beta>0$, then it's probability density function (pdf) is given by

$$
g(x)=\left(\alpha+\frac{\beta}{x^{2}}\right) \mathrm{e}^{\alpha x-\frac{\beta}{x}} \exp \left\{-\mathrm{e}^{\alpha x-\frac{\beta}{x}}\right\}, x>0
$$

while the cumulative distribution function (cdf) is given by

$$
G(x)=1-\exp \left\{-\mathrm{e}^{\alpha x-\frac{\beta}{x}}\right\}, x>0 .
$$

The survival function is given by the equation

$$
S(x)=1-G(x)=\exp \left\{-\mathrm{e}^{\alpha x-\frac{\beta}{x}}\right\}, x>0
$$

and the hazard rate function is

$$
h(x)=\left(\alpha+\frac{\beta}{x^{2}}\right) \mathrm{e}^{\alpha x-\frac{\beta}{x}} .
$$

In this article, a new generalization of the Flexible Weibull Extension (FWE) distribution called exponential flexible Weibull extension (EFWE) distribution is derived. Using the exponential generator applied to the odds ratio $\frac{1}{1-G(x)}$, such as the exponential Pareto distribution by AL-Kadim and Boshi [14], exponential lomax distribution by El-Bassiouny et al. [15]. If $X$ is a random variable and $G(x)$ is the baseline cumulative distribution function with probability density function $g(x)$ and the exponential cumulative distribution function is

$$
F(x ; \lambda)=1-\mathrm{e}^{-\lambda x}, \quad x \geq 0, \quad \lambda \geq 0 .
$$

By using Equation (5) and replacing the random variable $X$ with ratio $\frac{1}{1-G(x)}$. The cdf of exponential generalized distribution is defined by AL-Kadim and Boshi [14] and El-Bassiouny et al. [15]

$$
\begin{aligned}
F(x) & =\int_{0}^{\frac{1}{1-G(x)}} \lambda \mathrm{e}^{-\lambda t} \mathrm{~d} t \\
& =1-\exp \left\{-\lambda\left[\frac{1}{1-G(x)}\right]\right\}, x \geq 0, \lambda \geq 0,
\end{aligned}
$$

where $G(x)$ is a baseline cdf. Hence the pdf corresponding to Equation (6) is given as

$$
f(x)=\frac{\lambda \cdot g(x)}{[1-G(x)]^{2}} \cdot \exp \left\{-\lambda\left[\frac{1}{1-G(x)}\right]\right\} \text {. }
$$

This article is organized as follows. The cumulative function, density function and hazard function of the exponential flexible Weibull extension (EFWE) distribution are 
defined in Section 2. Some of statistical properties including, quantile function and simulation, the mode, median, the skewness and kurtosis and rth moments are presented in Sections 3. The moment generating function (mgf) is derived in Sections 4. The order statistics is determined in Section 5. The maximum likelihood estimation of the parameters is obtained in Section 6. Real data sets are analyzed in Section 7. Moreover, we discuss the results and compare it with existing distributions. Finally, we introduce the conclusion of our results.

\section{Definition of the EFWE Distribution}

We define in this section three parameters of the Exponential Flexible Weibull Extension EFWE $(\alpha, \beta, \lambda)$ distribution. Using $G(x)$ Equation (2) and $g(x)$ Equation (1) in Equation (6) and Equation (7) to obtain the cdf and pdf of EFWE distribution. The cumulative distribution function cdf of the Exponential Flexible Weibull Extension distribution (EFWE) is given by

$$
F(x ; \alpha, \beta, \lambda)=1-\exp \left\{-\lambda \mathrm{e}^{\mathrm{e} x-\frac{\beta}{x}}\right\}, x>0, \alpha, \beta, \lambda>0 .
$$

The pdf corresponding to Equation (8) is given by

$$
f(x ; \alpha, \beta, \lambda)=\lambda\left(\alpha+\frac{\beta}{x^{2}}\right) \mathrm{e}^{\alpha x-\frac{\beta}{x}} \mathrm{e}^{\mathrm{e}^{\alpha x-\frac{\beta}{x}}} \exp \left\{-\lambda \mathrm{e}^{\mathrm{e}^{\alpha x-\frac{\beta}{x}}}\right\},
$$

where $x>0$ and $\alpha, \beta>0$ are two additional shape parameters.

The survival function $S(x)$, hazard rate function $h(x)$, reversed hazard rate function $r(x)$ and cumulative hazard rate function $H(x)$ of $X \sim \operatorname{EFWE}(\alpha, \beta, \lambda)$ are given by

$$
\begin{gathered}
S(x ; \alpha, \beta, \lambda)=1-F(x ; \alpha, \beta, \lambda)=\exp \left\{-\lambda \mathrm{e}^{\mathrm{e}^{\alpha x-\frac{\beta}{x}}}\right\}, x>0, \\
h(x ; \alpha, \beta, \lambda)=\lambda\left(\alpha+\frac{\beta}{x^{2}}\right) \mathrm{e}^{\alpha x-\frac{\beta}{x}} \mathrm{e}^{\alpha x-\frac{\beta}{x}}, \\
r(x ; \alpha, \beta, \lambda)=\frac{\lambda\left(\alpha+\frac{\beta}{x^{2}}\right) \mathrm{e}^{\alpha x-\frac{\beta}{x}} \mathrm{e}^{\mathrm{e}^{\alpha x-\frac{\beta}{x}}} \mathrm{exp}\left\{-\lambda \mathrm{e}^{\mathrm{e}^{\alpha x-\frac{\beta}{x}}}\right\}}{1-\exp \left\{-\lambda \mathrm{e}^{\mathrm{e}^{\alpha x-\frac{\beta}{x}}}\right\}} \\
H(x ; \alpha, \beta, \lambda)=\int_{0}^{x} h(u) \mathrm{d} u=\lambda \exp \left\{\mathrm{e}^{\alpha x-\frac{\beta}{x}}\right\},
\end{gathered}
$$

respectively, $x>0$ and $\alpha, \beta, \lambda>0$.

Figures 1-6 display the cdf, pdf, survival function, hazard rate function, reversed hazard rate function and cumulative hazard rate function of the $\operatorname{EFWE}(\alpha, \beta, \lambda)$ distribution for some parameter values.

\section{Some Statistical Properties}

Some statistical properties for the EFWE distribution, such as quantile and simulation 


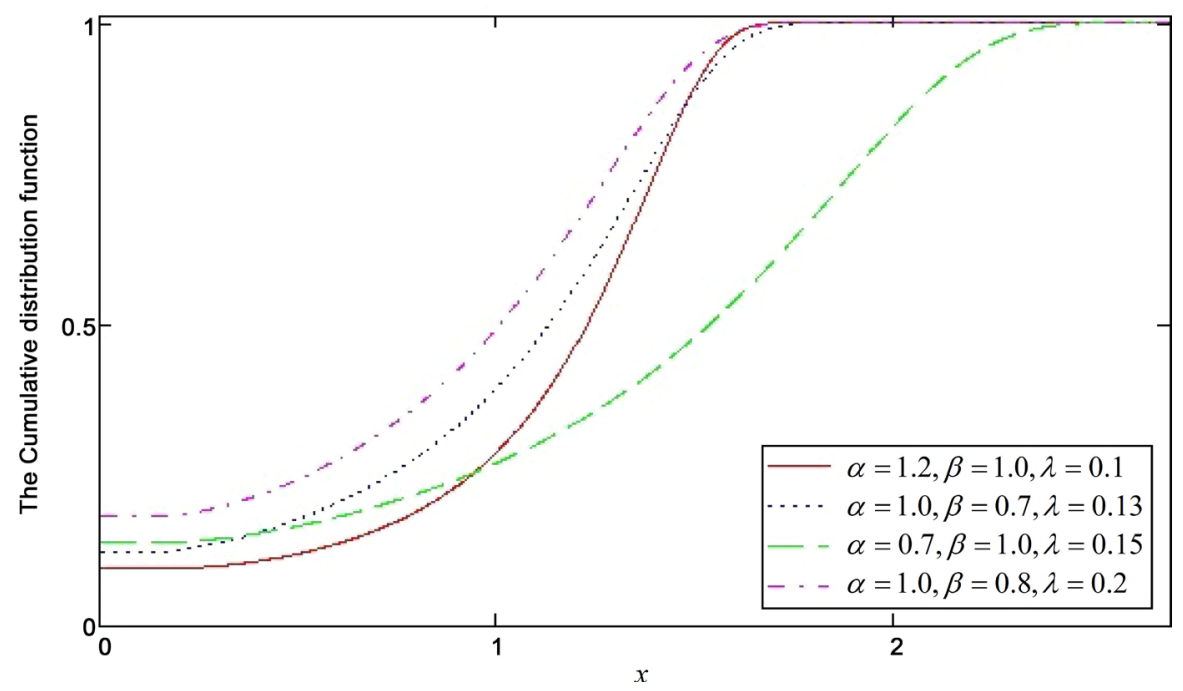

Figure 1. The cdf of the EFWE for different values of parameters.

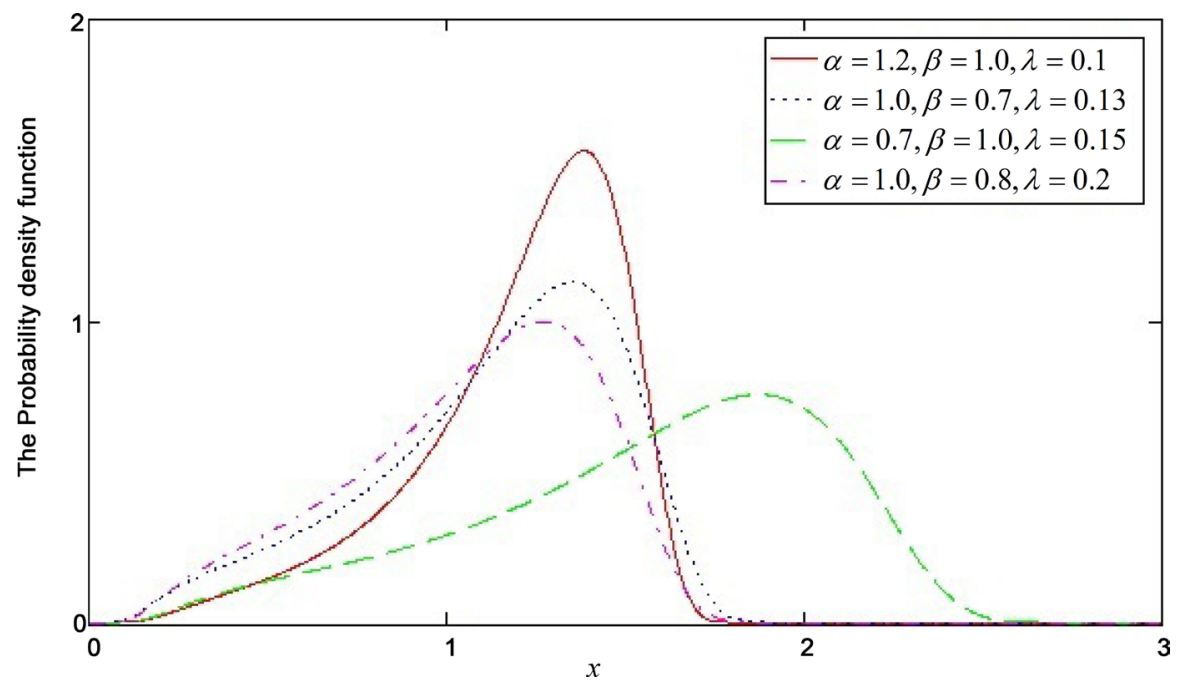

Figure 2. The pdf of the EFWE for different values of parameters.

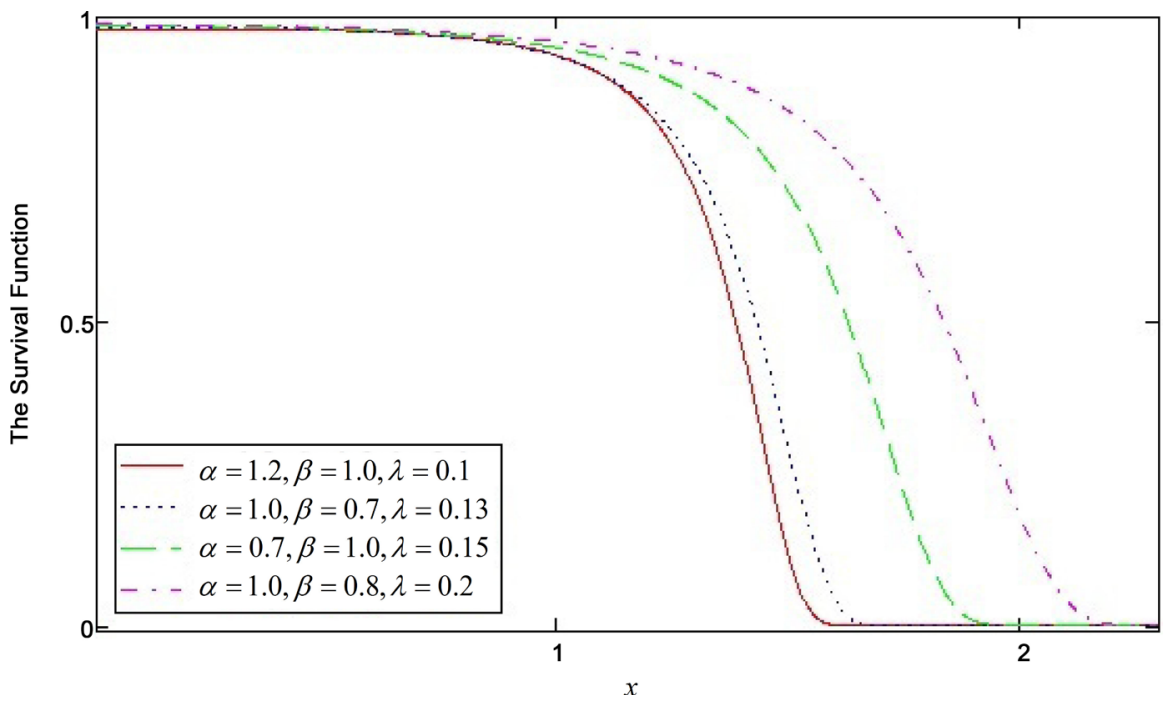

Figure 3. The $S(x)$ of the EFWE for different values of parameters. 


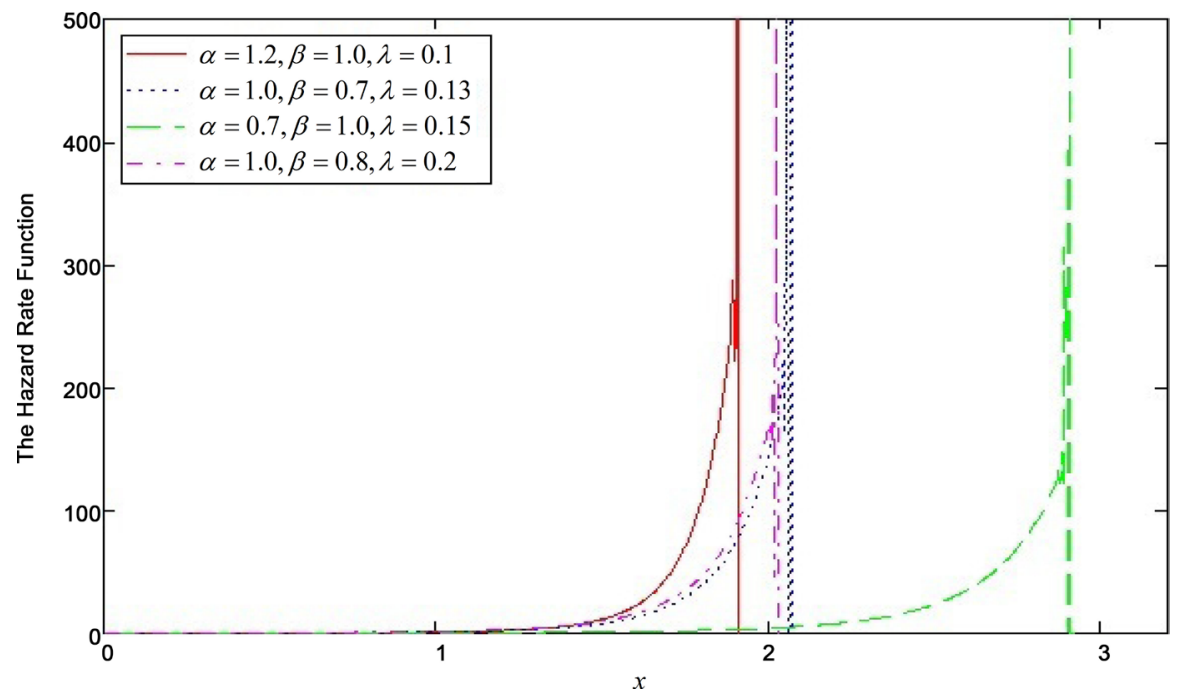

Figure 4. The $h(x)$ of the EFWE for different values of parameters.

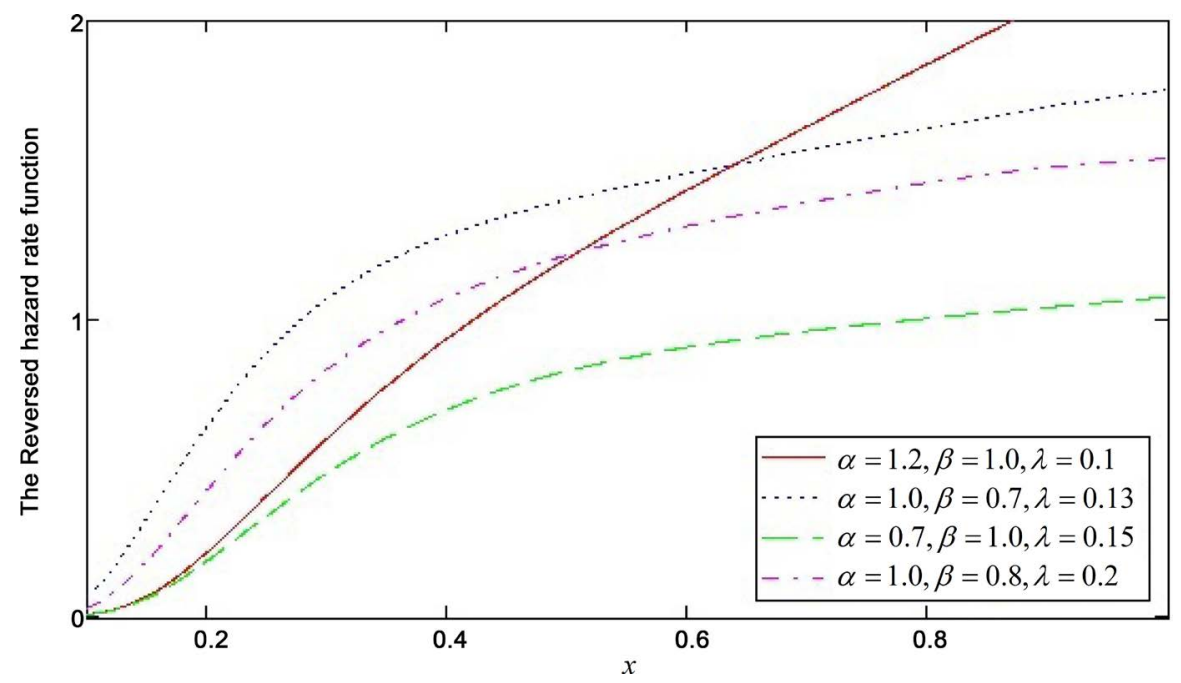

Figure 5. The $r(x)$ of the EFWE for different values of parameters.

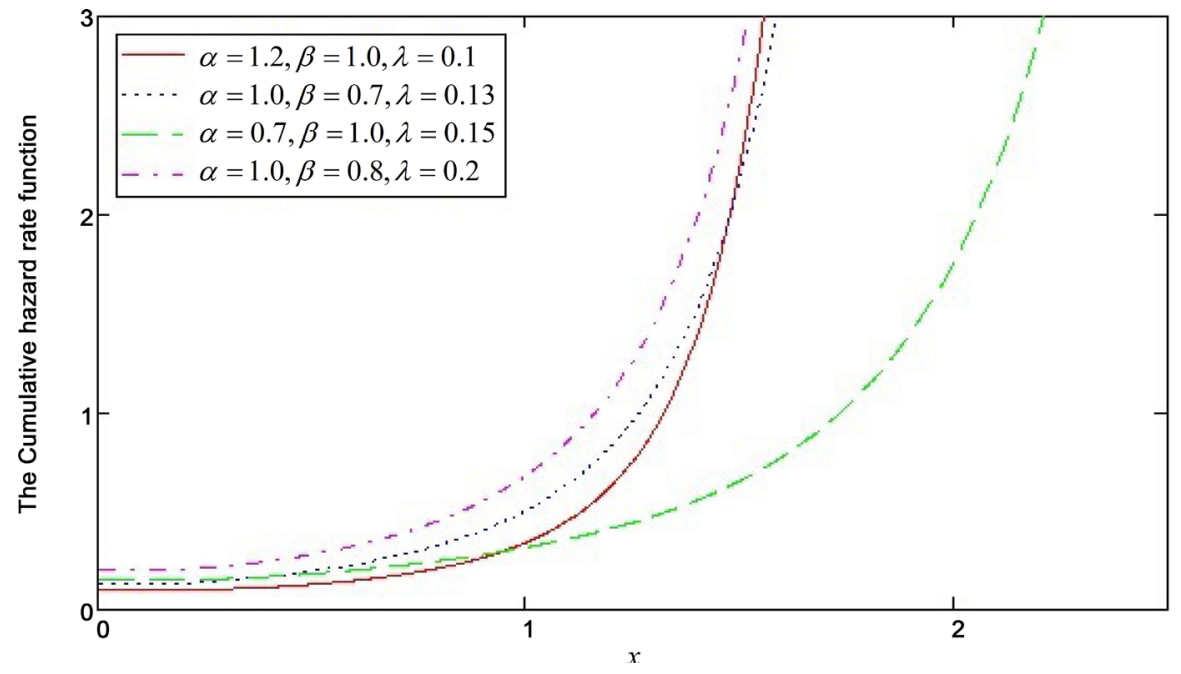

Figure 6. The $H(x)$ of the EFWE for different values of parameters. 
median, the mode, moments, the skewness and kurtosis are given as follows.

\subsection{The Quantile and Simulation}

Suppose the random variable $x_{q}$ is the quantile of the EFWE distribution given by

$$
F\left(x_{q}\right)=q, \quad 0<q<1 .
$$

Using the distribution function of EFWE distribution, from (8), we have

$$
\alpha x_{q}^{2}-k(q) x_{q}-\beta=0,
$$

where

$$
k(q)=\ln \left\{\ln \left(-\frac{\ln (1-q)}{\lambda}\right)\right\} .
$$

So, the simulation of the EFWE distribution random variable is straightforward. We obtain the random variable $X$ by solve the Equation (15) as follows form

$$
X=\frac{k(u) \pm \sqrt{k(u)^{2}+4 \alpha \beta}}{2 \alpha} .
$$

Since the median is $50 \%$ quantile then by setting $q=0.5$ in Equation (15), we can obtain the median $M$ of EFWE distribution.

\subsection{The Mode of EFWE}

The mode of the EFWE distribution can be obtained by differentiating its probability density function in Equation (9) with respect to $x$ and equaling it to zero. So the mode of the EFWE is the solution of the following equation

$$
\lambda \mathrm{e}^{\alpha x-\frac{\beta}{x}} \mathrm{e}^{\alpha x-\frac{\beta}{x}} \exp \left\{-\lambda \mathrm{e}^{\alpha x-\frac{\beta}{x}}\right\}\left[-\frac{2 \beta}{x^{3}}+\left(\alpha+\frac{\beta}{x^{2}}\right)\left(1+\mathrm{e}^{\alpha x-\frac{\beta}{x}}-\lambda \mathrm{e}^{\alpha x-\frac{\beta}{x}}\right)\right]=0
$$

The EFWE distribution has only one peak, then this distribution is a unimodal distribution. Figure 2 shows that Equation (18) has only one solution. It is difficult to get an explicit solution of Equation (18). Therefore, it can be solved numerically. Some values of median and mode for various values of the parameters $\alpha, \beta$ and $\lambda$ are calculated in Table 1.

\subsection{The Skewness and Kurtosis}

In this subsection, we can obtain the skewness and kurtosis based on the quantile

Table 1. The median and mode for EFWE $(\alpha, \beta, \lambda)$.

\begin{tabular}{ccccc}
\hline$\alpha$ & $\beta$ & $\lambda$ & median & mode \\
\hline 0.015 & 0.381 & 0.076 & 53.3576 & 10.6657 \\
0.158 & 0.158 & 0.273 & 0.801066 & 1.96923 \\
0.700 & 1.000 & 0.150 & 1.537340 & 1.87122 \\
1.000 & 0.700 & 0.130 & 1.132920 & 1.35312 \\
1.000 & 0.800 & 0.200 & 1.009750 & 1.27259 \\
1.200 & 1.000 & 0.100 & 1.228750 & 1.38465 \\
\hline
\end{tabular}


measures. The Bowely's skewness (Kenney and Keeping [16]) is given by

$$
S_{k}=\frac{q_{(0.75)}-2 q_{(0.5)}+q_{(0.25)}}{q_{(0.75)}-q_{(0.25)}}
$$

and the Moors Kurtosis (Moors [17]) can be obtained, based on octiles, as follows

$$
K_{u}=\frac{q_{(0.875)}-q_{(0.625)}-q_{(0.375)}+q_{(0.125)}}{q_{(0.75)}-q_{(0.25)}},
$$

where the $q_{(.)}$is quantile function.

\subsection{The Moments}

Here, we derive the rth moment for EFWE distribution in the next theorem

Theorem 1. If $X$ has EFWE $(\alpha, \beta, \lambda)$ distribution, then the rth moments of random variable $X$, is given by

$$
\mu_{r}^{\prime}=\sum_{i=0}^{\infty} \sum_{j=0}^{\infty} \sum_{k=0}^{\infty} \frac{(-1)^{i+k} \lambda^{i+1} \beta^{k}(i+1)^{j}(j+1)^{k}}{i ! j ! k !}\left[\frac{\Gamma(r-k+1)}{\alpha^{r-k}(j+1)^{r-k+1}}+\frac{\beta \Gamma(r-k-1)}{\alpha^{r-k-1}(j+1)^{r-k-1}}\right] .
$$

Proof. From the definition of the moments, we know that the rth moment of the random variable $X$ with the pdf $f(x)$ is given by

$$
\mu_{r}^{\prime}=\int_{0}^{\infty} x^{r} f(x ; \alpha, \beta, \lambda) \mathrm{d} x .
$$

Substituting from Equation (9) into Equation (22) we get

$$
\mu_{r}^{\prime}=\int_{0}^{\infty} x^{r} \lambda\left(\alpha+\frac{\beta}{x^{2}}\right) \mathrm{e}^{\alpha x-\frac{\beta}{x}} \mathrm{e}^{\mathrm{e}^{\alpha x-\frac{\beta}{x}}} \exp \left\{-\lambda \mathrm{e}^{\mathrm{e}^{\alpha x-\frac{\beta}{x}}}\right\} \mathrm{d} x,
$$

the expansion of $\exp \left\{-\lambda \mathrm{e}^{\mathrm{e}^{\alpha x-\frac{\beta}{x}}}\right\}$ is

$$
\mathrm{e}^{-\lambda \mathrm{e}^{\alpha x-\frac{\beta}{x}}}=\sum_{i=0}^{\infty} \frac{(-1)^{i} \lambda^{i}}{i !} \mathrm{e}^{i \mathrm{e}^{\alpha x-\frac{\beta}{x}}},
$$

then we have

$$
\mu_{r}^{\prime}=\sum_{i=0}^{\infty} \frac{(-1)^{i} \lambda^{i+1}}{i !} \int_{0}^{\infty} x^{r}\left(\alpha+\frac{\beta}{x^{2}}\right) \mathrm{e}^{\alpha x-\frac{\beta}{x}} \mathrm{e}^{(i+1) \mathrm{e}^{\alpha x-\frac{\beta}{x}}} \mathrm{~d} x,
$$

using series expansion of $\mathrm{e}^{(i+1) \mathrm{e}^{\alpha x-\frac{\beta}{x}}}$,

$$
\mathrm{e}^{(i+1) \mathrm{e}^{\alpha x-\frac{\beta}{x}}}=\sum_{j=0}^{\infty} \frac{(i+1)^{j}}{j !} \mathrm{e}^{j\left(\alpha x-\frac{\beta}{x}\right)},
$$

hence, we obtain

$$
\begin{aligned}
\mu_{r}^{\prime} & =\sum_{i=0}^{\infty} \sum_{j=0}^{\infty} \frac{(-1)^{i} \lambda^{i+1}(i+1)^{j}}{i ! j !} \int_{0}^{\infty} x^{r}\left(\alpha+\beta x^{-2}\right) \mathrm{e}^{(j+1)\left(\alpha x-\frac{\beta}{x}\right)} \mathrm{d} x, \\
& =\sum_{i=0}^{\infty} \sum_{j=0}^{\infty} \frac{(-1)^{i} \lambda^{i+1}(i+1)^{j}}{i ! j !} \int_{0}^{\infty} x^{r}\left(\alpha+\beta x^{-2}\right) \mathrm{e}^{(j+1) \alpha x} \mathrm{e}^{-(j+1) \frac{\beta}{x}} \mathrm{~d} x,
\end{aligned}
$$


using series expansion of $\mathrm{e}^{-(j+1) \frac{\beta}{x}}$,

$$
\mathrm{e}^{-(j+1) \frac{\beta}{x}}=\sum_{k=0}^{\infty} \frac{(-1)^{k}(j+1)^{k} \beta^{k}}{k !} x^{-k},
$$

we have

$$
\begin{aligned}
\mu_{r}^{\prime}= & \sum_{i=0}^{\infty} \sum_{j=0}^{\infty} \sum_{k=0}^{\infty} \frac{(-1)^{i+k} \lambda^{i+1} \beta^{k}(i+1)^{j}(j+1)^{k}}{i ! j ! k !} \int_{0}^{\infty} x^{r-k}\left(\alpha+\frac{\beta}{x^{2}}\right) \mathrm{e}^{(j+1) \alpha x} \mathrm{~d} x, \\
= & \sum_{i=0}^{\infty} \sum_{j=0 k=0}^{\infty} \sum^{\infty} \frac{(-1)^{i+k} \lambda^{i+1} \beta^{k}(i+1)^{j}(j+1)^{k}}{i ! j ! k !}\left[\int_{0}^{\infty} \alpha x^{r-k} \mathrm{e}^{(j+1) \alpha x} \mathrm{~d} x\right. \\
& \left.+\int_{0}^{\infty} \beta x^{r-k-2} \mathrm{e}^{(j+1) \alpha x} \mathrm{~d} x\right],
\end{aligned}
$$

using the definition of the gamma function (Zwillinger [18]), in the follows form,

$$
\Gamma(z)=x^{z} \int_{0}^{\infty} \mathrm{e}^{t x} t^{z-1} \mathrm{~d} t, \quad z, x,>0 .
$$

Finally, the rth moment of EFWE distribution is obtained in the form

$$
\mu_{r}^{\prime}=\sum_{i=0}^{\infty} \sum_{j=0}^{\infty} \sum_{k=0}^{\infty} \frac{(-1)^{i+k} \lambda^{i+1} \beta^{k}(i+1)^{j}(j+1)^{k}}{i ! j ! k !} \times\left[\frac{\Gamma(r-k+1)}{\alpha^{r-k}(j+1)^{r-k+1}}+\frac{\beta \Gamma(r-k-1)}{\alpha^{r-k-1}(j+1)^{r-k-1}}\right] .
$$

This completes the proof.

\section{The Moment Generating Function}

The moment generating function (mgf) of the EFWE distribution is given by theorem 2 .

Theorem 2. If $X$ is a random variable from $\operatorname{EFWE}(\alpha, \beta, \lambda)$ distribution, then its moment generating function is

$$
\begin{aligned}
M_{X}(t)= & \sum_{i=0}^{\infty} \sum_{j=0}^{\infty} \sum_{k=0}^{\infty} \sum_{r=0}^{\infty} \frac{(-1)^{i+k} \lambda^{i+1} \beta^{k}(i+1)^{j}(j+1)^{k} t^{r}}{i ! j ! k ! r !} \\
& \times\left[\frac{\Gamma(r-k+1)}{\alpha^{r-k}(j+1)^{r-k+1}}+\frac{\beta \Gamma(r-k-1)}{\alpha^{r-k-1}(j+1)^{r-k-1}}\right] .
\end{aligned}
$$

Proof. The moment generating function (mgf) of the random variable $X$ with the pdf $f(x)$ is

$$
M_{X}(t)=\int_{0}^{\infty} \mathrm{e}^{t x} f(x) \mathrm{d} x
$$

using series expansion of $\mathrm{e}^{t x}$, we obtain

$$
M_{X}(t)=\sum_{r=0}^{\infty} \frac{t^{r}}{r !} \int_{0}^{\infty} x^{r} f(x) \mathrm{d} x .=\sum_{r=0}^{\infty} \frac{t^{r}}{r !} \mu_{r}^{\prime} .
$$

Substituting from Equation (21) into Equation (25) we obtain the moment generating function (mgf) of EFWE distribution in the form

$$
\begin{aligned}
M_{X}(t)= & \sum_{i=0}^{\infty} \sum_{j=0}^{\infty} \sum_{k=0}^{\infty} \sum_{r=0}^{\infty} \frac{(-1)^{i+k} \lambda^{i+1} \beta^{k}(i+1)^{j}(j+1)^{k} t^{r}}{i ! j ! k ! r !} \\
& \times\left[\frac{\Gamma(r-k+1)}{\alpha^{r-k}(j+1)^{r-k+1}}+\frac{\beta \Gamma(r-k-1)}{\alpha^{r-k-1}(j+1)^{r-k-1}}\right] .
\end{aligned}
$$


This completes the proof.

\section{The Order Statistics}

In this section, we derive closed form expressions for the probability density function pdf of the $r$ th order statistic of the EFWE distribution. Suppose $X_{1: n}, X_{2: n}, \cdots, X_{n: n}$ denote the order statistics obtained from a random sample $X_{1}, X_{2}, \cdots, X_{n}$ which taken from a continuous population with cumulative distribution function $F(x ; \varphi)$ and probability density function $f(x ; \varphi)$, then the pdf of $X_{r: n}$ is as follows

$$
f_{r: n}(x ; \varphi)=\frac{1}{B(r, n-r+1)}[F(x ; \varphi)]^{r-1}[1-F(x ; \varphi)]^{n-r} f(x ; \varphi),
$$

where $f(x ; \varphi)$ and $F(x ; \varphi)$ are the probability density function and cumulative distribution function of EFWE $(\alpha, \beta, \lambda)$ distribution given by Equation (9) and Equation (8) respectively, $B(.,$.$) is the Beta function and \varphi=(\alpha, \beta, \lambda)$, also we define first order statistics $X_{1: n}=\min \left(X_{1}, X_{2}, \cdots, X_{n}\right)$, and the last order statistics as $X_{n: n}=\max \left(X_{1}, X_{2}, \cdots, X_{n}\right)$, where $0<F(x ; \varphi)<1$ for all $x>0$. Using the binomial expansion of $[1-F(x ; \varphi)]^{n-r}$

$$
[1-F(x ; \varphi)]^{n-r}=\sum_{i=0}^{n-r}\left(\begin{array}{c}
n-r \\
i
\end{array}\right)(-1)^{i}[F(x ; \varphi)]^{i} .
$$

Substituting from Equation (27) into Equation (26), we have

$$
f_{r: n}(x ; \varphi)=\frac{1}{B(r, n-r+1)} f(x ; \varphi) \sum_{i=0}^{n-r}\left(\begin{array}{c}
n-r \\
i
\end{array}\right)(-1)^{i}[F(x ; \varphi)]^{i+r-1} .
$$

Substituting from Equation (8) and Equation (9) into Equation (28), we obtain

$$
f_{r: n}(x ; \varphi)=\sum_{i=0}^{n-r} \frac{(-1)^{i} n !}{i !(r-1) !(n-r-i) !}[F(x, \varphi)]^{i+r-1} f(x ; \varphi) .
$$

Relation (29) shows that $f_{r: n}(x ; \varphi)$ is the weighted average of the Exponential Flexible Weibull Extension distribution with different shape parameters.

\section{Parameters Estimation}

In this section, point and interval estimation of the unknown parameters of the EFWE distribution are derived by using the method of maximum likelihood based on a complete sample.

\subsection{Maximum Likelihood Estimation}

Let $x_{1}, x_{2}, \cdots, x_{n}$ denote a random sample of complete data from the EFWE distribution. The Likelihood function is given as

$$
L=\prod_{i=1}^{n} f\left(x_{i} ; \alpha, \beta, \lambda\right)
$$

substituting from (9) into (30), we have

$$
L=\prod_{i=1}^{n} \lambda\left(\alpha+\frac{\beta}{x_{i}^{2}}\right) \mathrm{e}^{\alpha x_{i}-\frac{\beta}{x_{i}}} \mathrm{e}^{\alpha x_{i}-\frac{\beta}{x_{i}}} \exp \left\{-\lambda \mathrm{e}^{\alpha x^{-\frac{\beta}{-}} \frac{\beta}{x_{i}}}\right\} .
$$


The log-likelihood function is

$$
\mathcal{L}=n \ln (\lambda)+\sum_{i=1}^{n} \ln \left(\alpha+\frac{\beta}{x_{i}^{2}}\right)+\sum_{i=1}^{n}\left(\alpha x_{i}-\frac{\beta}{x_{i}}\right)+\sum_{i=1}^{n} \mathrm{e}^{\alpha x_{i}-\frac{\beta}{x_{i}}}-\lambda \sum_{i=1}^{n} \mathrm{e}^{\alpha x_{i}-\frac{\beta}{x_{i}}} .
$$

The maximum likelihood estimation of the parameters are obtained by differentiating the log-likelihood function $\mathcal{L}$ with respect to the parameters $\alpha, \beta$ and $\lambda$ and setting the result to zero, as follows

$$
\begin{aligned}
& \frac{\partial \mathcal{L}}{\partial \alpha}=\sum_{i=1}^{n} \frac{x_{i}^{2}}{\beta+\alpha x_{i}^{2}}+\sum_{i=1}^{n} x_{i}+\sum_{i=1}^{n} x_{i} \mathrm{e}^{\alpha x_{i}-\frac{\beta}{x_{i}}}-\lambda \sum_{i=1}^{n} x_{i} \mathrm{e}^{\alpha x_{i}-\frac{\beta}{x_{i}}} \mathrm{e}^{\alpha x_{i}-\frac{\beta}{x_{i}}}=0, \\
& \frac{\partial \mathcal{L}}{\partial \beta}=\sum_{i=1}^{n} \frac{1}{\beta+\alpha x_{i}^{2}}-\sum_{i=1}^{n} \frac{1}{x_{i}}-\sum_{i=1}^{n} \frac{1}{x_{i}} \mathrm{e}^{\alpha x_{i}-\frac{\beta}{x_{i}}}+\lambda \sum_{i=1}^{n} \frac{1}{x_{i}} \mathrm{e}^{\alpha x_{i}-\frac{\beta}{x_{i}}} \mathrm{e}^{\alpha x_{i}-\frac{\beta}{x_{i}}}=0, \\
& \frac{\partial \mathcal{L}}{\partial \lambda}=\frac{n}{\lambda}-\sum_{i=1}^{n} \mathrm{e}^{\alpha x_{i}-\frac{\beta}{x_{i}}}=0 .
\end{aligned}
$$

The MLEs can be obtained by solving the nonlinear Equations (32)-(34), numerically for $\alpha, \beta$ and $\lambda$ by using Mathcad program.

\subsection{Asymptotic Confidence Bounds}

The asymptotic confidence intervals can be obtained when the parameters $\alpha, \beta$ and $\lambda$ are positive as the maximum likelihood estimations of the unknown parameters $(\alpha, \beta, \lambda)$ but can't be obtained in closed forms. So, by using variance covariance matrix $\boldsymbol{I}^{-1}$ see (Lawless [19]), where the $\boldsymbol{I}^{-1}$ is inverse of the observed information matrix which defined as follows

$$
\boldsymbol{I}^{-1}=\left(\begin{array}{rrr}
-\frac{\partial^{2} \mathcal{L}}{\partial \alpha^{2}} & -\frac{\partial^{2} \mathcal{L}}{\partial \alpha \partial \beta} & -\frac{\partial^{2} \mathcal{L}}{\partial \alpha \partial \lambda} \\
-\frac{\partial^{2} \mathcal{L}}{\partial \beta \partial \alpha} & -\frac{\partial^{2} \mathcal{L}}{\partial \beta^{2}} & -\frac{\partial^{2} \mathcal{L}}{\partial \beta \partial \lambda} \\
-\frac{\partial^{2} \mathcal{L}}{\partial \lambda \partial \alpha} & -\frac{\partial^{2} \mathcal{L}}{\partial \lambda \partial \beta} & -\frac{\partial^{2} \mathcal{L}}{\partial \lambda^{2}}
\end{array}\right)^{-1}=\left(\begin{array}{lll}
\operatorname{var}(\hat{\alpha}) & \operatorname{cov}(\hat{\alpha}, \hat{\beta}) & \operatorname{cov}(\hat{\alpha}, \hat{\lambda}) \\
\operatorname{cov}(\hat{\beta}, \hat{\alpha}) & \operatorname{var}(\hat{\beta}) & \operatorname{cov}(\hat{\beta}, \hat{\lambda}) \\
\operatorname{cov}(\hat{\lambda}, \hat{\alpha}) & \operatorname{cov}(\hat{\lambda}, \hat{\beta}) & \operatorname{var}(\hat{\lambda})
\end{array}\right),
$$

where

$$
\begin{aligned}
& \frac{\partial^{2} \mathcal{L}}{\partial \alpha^{2}}=-\sum_{i=1}^{n} \frac{x_{i}^{4}}{\left(\beta+\alpha x_{i}^{2}\right)^{2}}+\sum_{i=1}^{n} x_{i}^{2} \mathrm{e}^{\alpha x_{i}-\frac{\beta}{x_{i}}}-\lambda \sum_{i=1}^{n} x_{i}^{2} \mathrm{e}^{\alpha x_{i}-\frac{\beta}{x_{i}}} \mathrm{e}^{\alpha x_{i}-\frac{\beta}{x_{i}}}\left[1+\mathrm{e}^{\alpha x_{i}-\frac{\beta}{x_{i}}}\right], \\
& \frac{\partial^{2} \mathcal{L}}{\partial \alpha \partial \beta}=-\sum_{i=1}^{n} \frac{x_{i}^{2}}{\left(\beta+\alpha x_{i}^{2}\right)^{2}}-\sum_{i=1}^{n} \mathrm{e}^{\alpha x_{i}-\frac{\beta}{x_{i}}}+\lambda \sum_{i=1}^{n} \mathrm{e}^{\alpha x_{i}-\frac{\beta}{x_{i}}} \mathrm{e}^{\alpha x_{i}-\frac{\beta}{x_{i}}}\left[1+\mathrm{e}^{\alpha x_{i}-\frac{\beta}{x_{i}}}\right] \text {, } \\
& \frac{\partial^{2} \mathcal{L}}{\partial \alpha \partial \lambda}=-\sum_{i=1}^{n} X_{i} \mathrm{e}^{\alpha x_{i}-\frac{\beta}{x_{i}}} \mathrm{e}^{\alpha x_{i}-\frac{\beta}{x_{i}}}, \\
& \frac{\partial^{2} \mathcal{L}}{\partial \beta^{2}}=-\sum_{i=1}^{n} \frac{1}{\left(\beta+\alpha x_{i}^{2}\right)^{2}}+\sum_{i=1}^{n} \frac{1}{x_{i}^{2}} \mathrm{e}^{\alpha x_{i}-\frac{\beta}{x_{i}}}-\lambda \sum_{i=1}^{n} \frac{1}{x_{i}^{2}} \mathrm{e}^{\alpha x_{i}-\frac{\beta}{x_{i}}} \mathrm{e}^{\alpha x_{i}-\frac{\beta}{x_{i}}}\left[1+\mathrm{e}^{\alpha x_{i}-\frac{\beta}{x_{i}}}\right] \text {, } \\
& \frac{\partial^{2} \mathcal{L}}{\partial \beta \partial \lambda}=\sum_{i=1}^{n} \frac{1}{x_{i}} \mathrm{e}^{\alpha x_{i}-\frac{\beta}{x_{i}}} \mathrm{e}^{\alpha x_{i} \frac{\beta}{x_{i}}},
\end{aligned}
$$




$$
\frac{\partial^{2} \mathcal{L}}{\partial \lambda^{2}}=-\frac{n}{\lambda^{2}}
$$

Furthermore, the $(1-\delta) 100 \%$ confidence intervals of the parameters $(\alpha, \beta, \lambda)$ can be obtained by using variance matrix as the following forms

$$
\hat{\alpha} \pm Z_{\frac{\delta}{2}} \sqrt{\operatorname{var}(\hat{\alpha})}, \quad \hat{\beta} \pm Z_{\frac{\delta}{2}} \sqrt{\operatorname{var}(\hat{\beta})}, \quad \hat{\lambda} \pm Z_{\frac{\delta}{2}} \sqrt{\operatorname{var}(\hat{\lambda})},
$$

where $\delta>0$, and $Z_{\frac{\delta}{2}}$ denote the upper $\left(\frac{\delta}{2}\right)$-th percent of the standard normal distribution.

\section{Application}

In this application, we will analysis a real data set given by Aarset [22], see Table 2, using the $\operatorname{EFWE}(\alpha, \beta, \lambda)$ distribution and compare it with the other fitted distributions like a flexible Weibull extension (FWE) distribution, Weibull (W) distribution, linear failure rate (LFR) distribution, Exponentiated Weibull (EW) distribution, generalized linear failure rate (GLFR) distribution and Exponentiated Flexible Weibull (EFW) distribution by using Kolmogorov Smirnov (K-S) statistic, P-value, Akaike information criterion (AIC), as well as Akaike Information Criterion with correction (AICC), see [20] and also Bayesian information criterion (BIC), see [21], values.

Table 3 gives the maximum likelihood estimations of parameters for EFWE distribution, the value of K-S Statistics and P-value. As well the values of the log-likelihood functions, AIC, AICC and BIC are in Table 4.

From Table 3 and Table 4 we note that the EFWE distribution with three parameters gives a better fit than the previous models distributions. It has the largest log-likelihood

Table 2. Life time of 50 devices, see Aarset [22].

\begin{tabular}{cccccccccc}
\hline 0.1 & 0.2 & 1 & 1 & 1 & 1 & 1 & 2 & 3 & 6 \\
7 & 11 & 12 & 18 & 18 & 18 & 18 & 18 & 21 & 32 \\
36 & 40 & 45 & 46 & 47 & 50 & 55 & 60 & 63 & 63 \\
67 & 67 & 67 & 67 & 72 & 75 & 79 & 82 & 82 & 83 \\
84 & 84 & 84 & 85 & 85 & 85 & 85 & 85 & 86 & 86 \\
\hline
\end{tabular}

Table 3. The maximum likelihood estimations and K-S of parameters for Aarset data [22].

\begin{tabular}{cccc}
\hline Model & MLE of the parameters & K-S & P-value \\
\hline FW $(\alpha, \beta)$ & $\hat{\alpha}=0.0122, \hat{\beta}=0.7002$ & 0.4386 & $4.29 \times 10^{-9}$ \\
W $(\alpha, \beta)$ & $\hat{\alpha}=44.913, \hat{\beta}=0.949$ & 0.2397 & 0.0052 \\
LFR $(a, b)$ & $\hat{a}=0.014, \hat{b}=2.4 \times 10^{-4}$ & 0.1955 & 0.0370 \\
EW $(\alpha, \beta, \gamma)$ & $\hat{\alpha}=91.023, \hat{\beta}=4.69, \hat{\gamma}=0.164$ & 0.1841 & 0.0590 \\
GLFR $(a, b, c)$ & $\hat{a}=0.0038, \hat{b}=3.04 \times 10^{-4}, \hat{c}=0.533$ & 0.1620 & 0.1293 \\
$\operatorname{EFW~}(\alpha, \beta, \theta)$ & $\hat{\alpha}=0.0147, \hat{\beta}=0.133, \hat{\theta}=4.22$ & 0.1433 & 0.2617 \\
$\operatorname{EFWE~}(\alpha, \beta, \lambda)$ & $\hat{\alpha}=0.015, \hat{\beta}=0.381, \hat{\lambda}=0.076$ & 0.1387 & 0.2719 \\
\hline
\end{tabular}


Table 4. The log-likelihood function, AIC, AICC and BIC values of distributions for Aarset data [22].

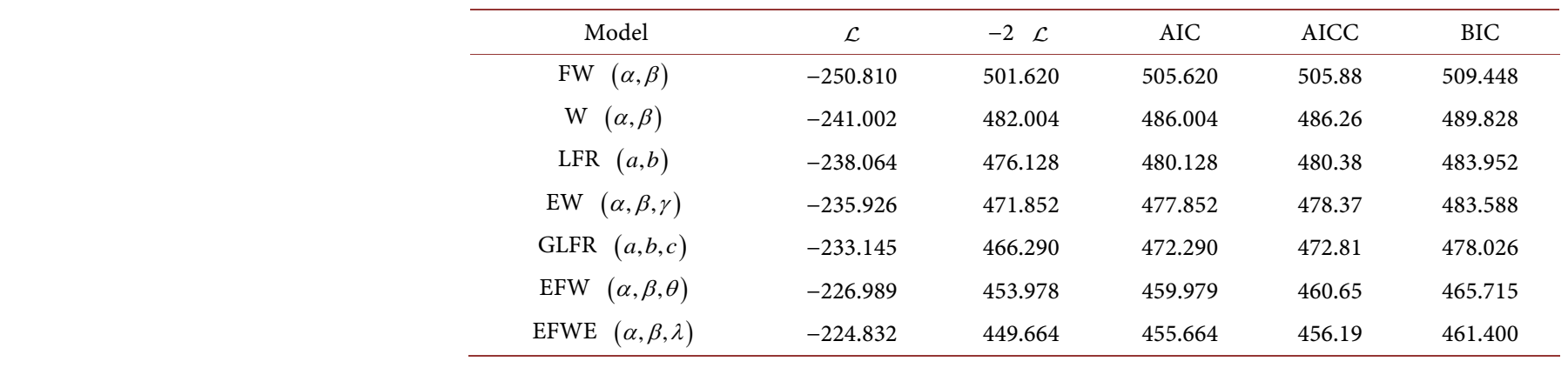
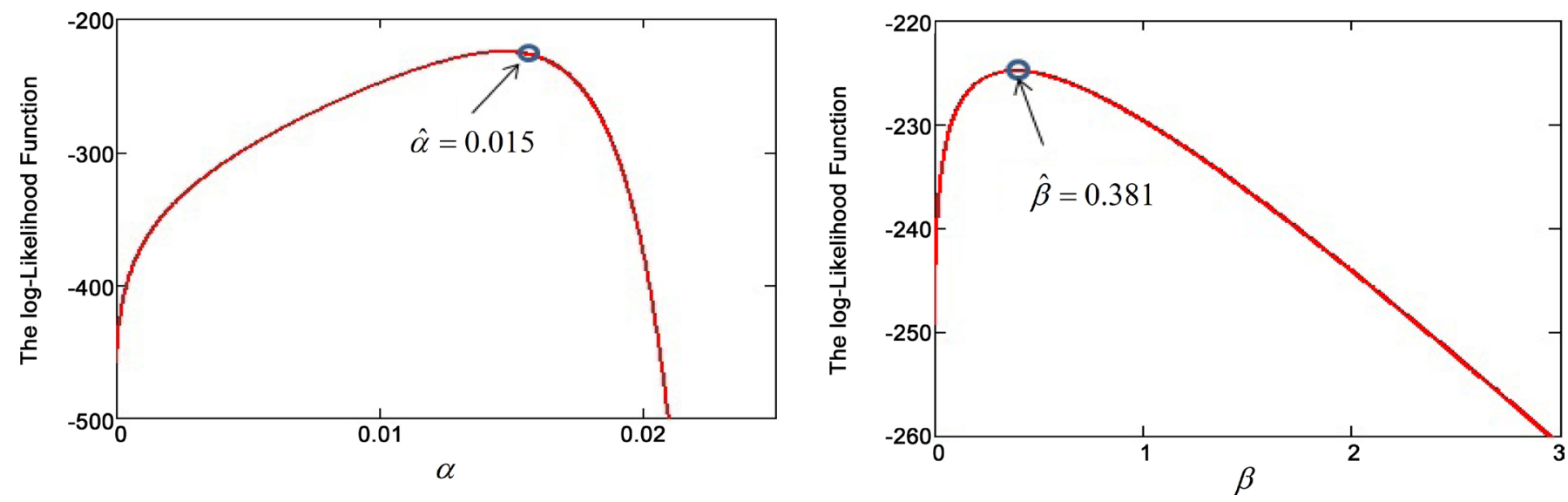

Figure 7. Profile of the log-likelihood for $\alpha, \beta$.

function, and also P-value, on the other hand the smallest K-S, AIC, AICC and BIC values from among those considered in this article.

Substituting the maximum likelihood estimations of the unknown parameters $\alpha, \beta$ and $\lambda$ into Equation (35), we have the estimation of the variance covariance matrix as follows

$$
\boldsymbol{I}_{0}^{-1}=\left(\begin{array}{lll}
1.11 \times 10^{-6} & -1.175 \times 10^{-5} & -2.187 \times 10^{-5} \\
-1.175 \times 10^{-5} & 0.021 & 3.275 \times 10^{-4} \\
-2.187 \times 10^{-5} & 3.275 \times 10^{-4} & 5.469 \times 10^{-4}
\end{array}\right)
$$

The approximate 95\% two sided confidence intervals of the unknown parameters $\alpha, \beta$ and $\lambda$ are $[0.013,0.017],[0.1,0.662]$ and $[0.03,0.122]$, respectively.

From, Figure 7 and Figure 8 we note that the likelihood function have unique solution.

Figure 9 represents the estimation for the survival function $S(x)$, by using the Kaplan-Meier method and its fitted parametric estimations when the distribution is assumed to be FW, W, LFR, EW, GLFR, EFW and EFWE are computed and plotted in the following shape.

Figure 10 and Figure 11 give the form of the hazard rate $h(x)$ and cumulative distribution function for the FW, W, LFR, EW, GLFR, EFW and EFWE which are used to fit the data when the unknown parameters included in each distribution are replaced by their maximum likelihood estimation. 


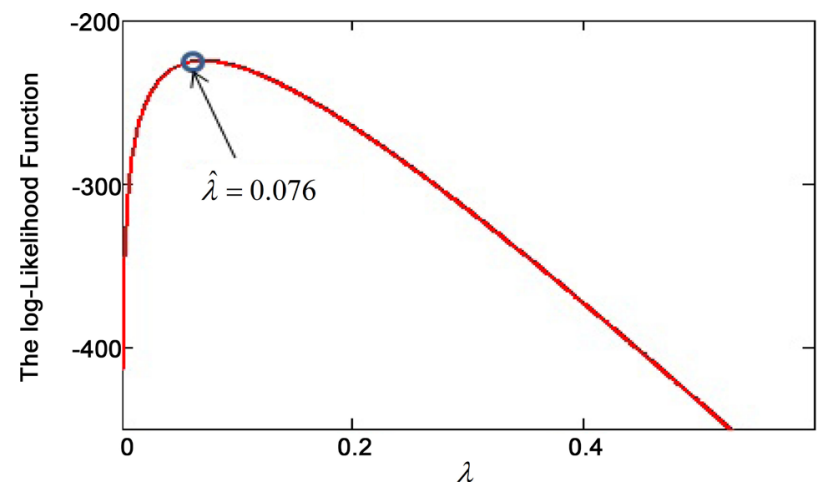

Figure 8. Profile of the log-likelihood for $\lambda$.

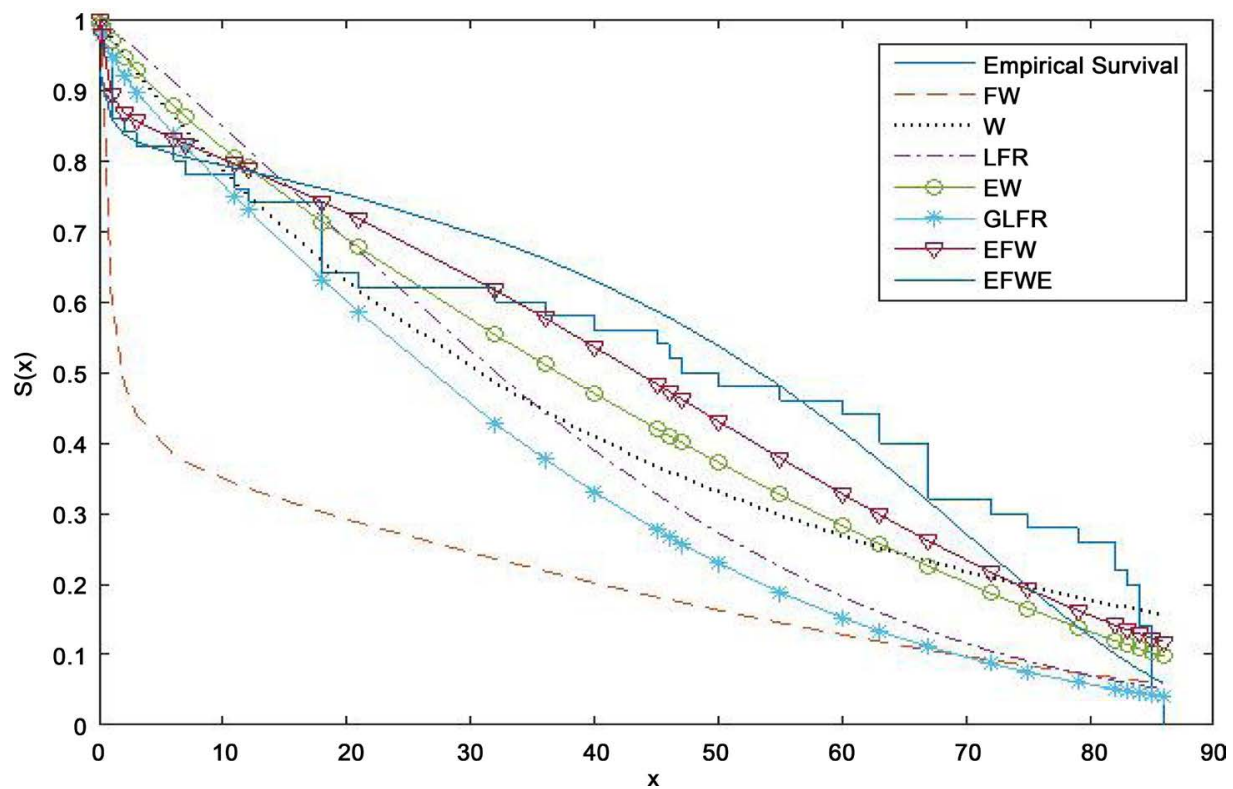

Figure 9. The Kaplan-Meier estimate of the survival function $S(x)$ for Aarset data [22].

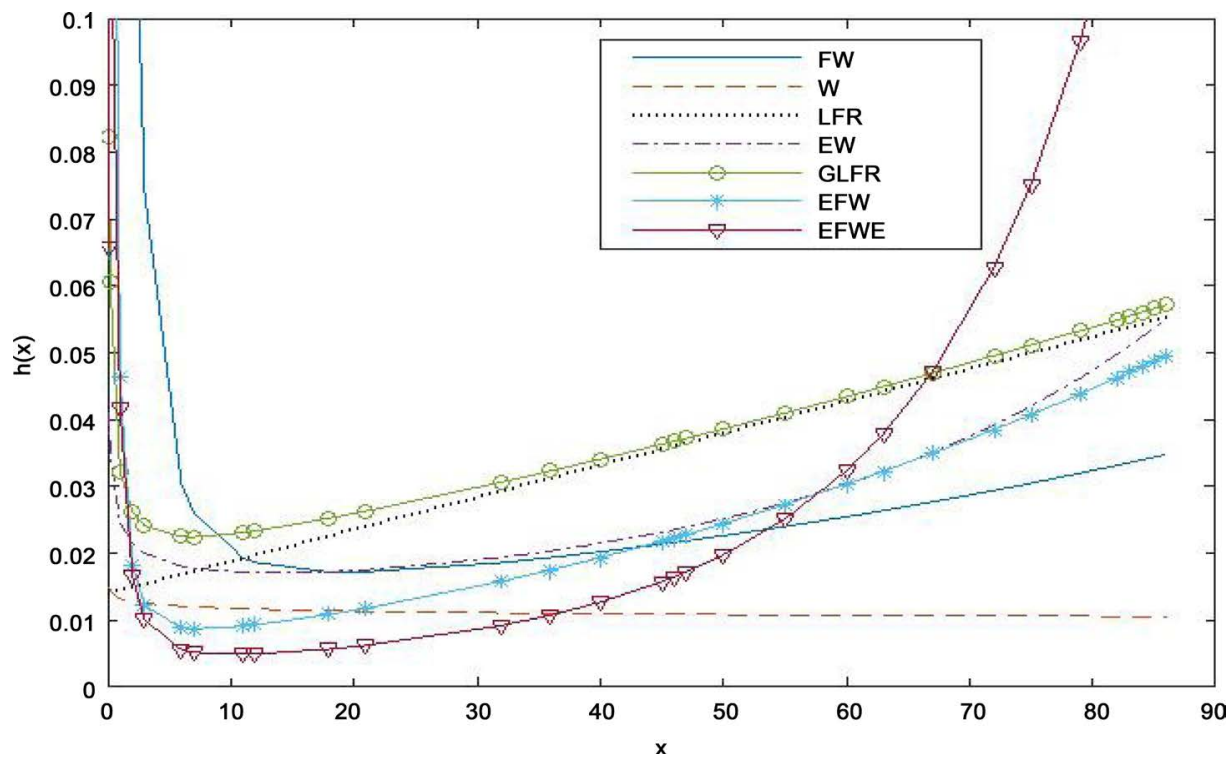

Figure 10. Fitted hazard rate function $h(x)$ for Aarset data [22]. 


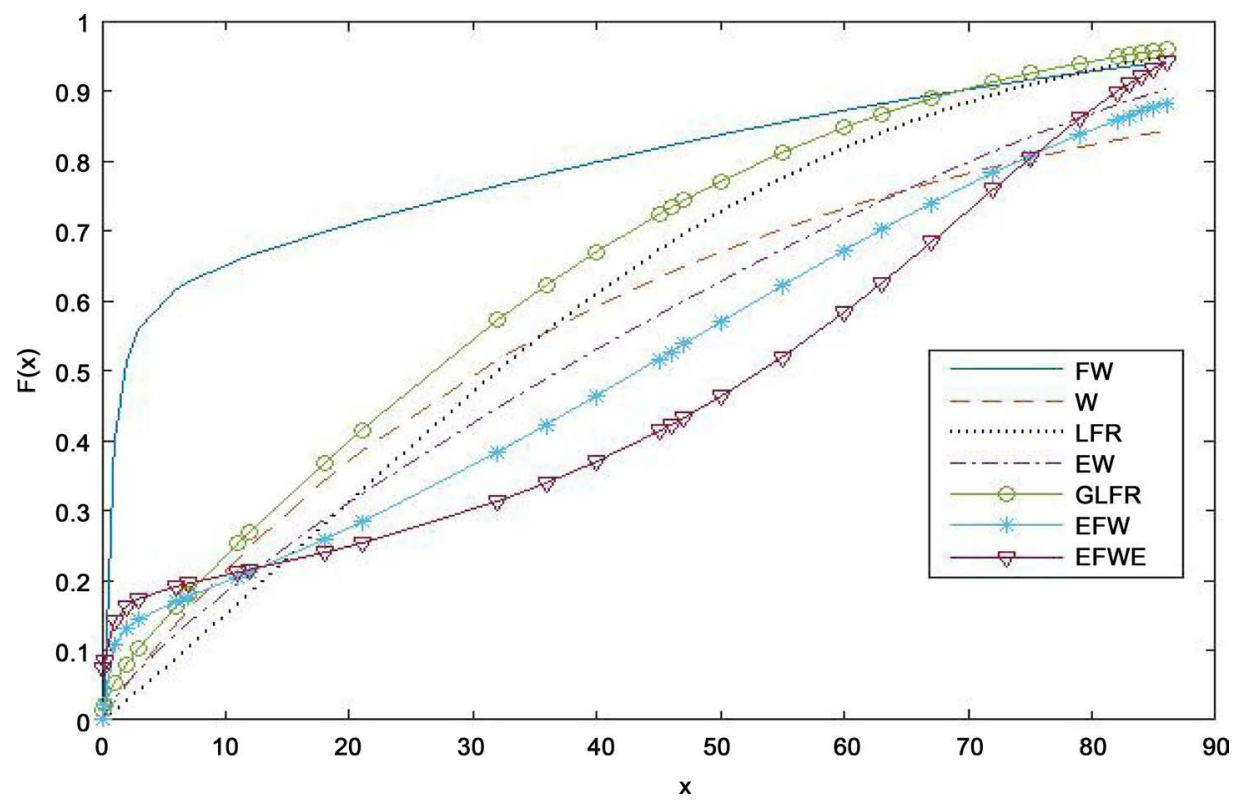

Figure 11. Fitted cumulative distribution function $F(x)$ for Aarset data [22].

\section{Conclusion}

We propose a new distribution, based on the exponential generalized distribution. The general idea is to add parameter to a flexible Weibull extension FWE distribution, this new distribution is called the exponential flexible Weibull extension EFWE. Its definition and some of statistical properties are studied. We use the maximum likelihood method for estimating parameters. Finally, the advantage of the EFWE distribution is concluded by an application using real data. Moreover, it is shown that the exponential flexible Weibull extension EFWE distribution fits better than existing modifications of the Weibull and flexible Weibull distributions.

\section{References}

[1] Weibull, W.A. (1951) Statistical Distribution Function of Wide Applicability. Journal of Applied Mechanics, 18, 293-296.

[2] Xie, M. and Lai, C.D. (1995) Reliability Analysis Using an Additive Weibull Model with Bathtub-Shaped Failure Ratefunction. Reliability Engineering System Safety, 52, 87-93. https://doi.org/10.1016/0951-8320(95)00149-2

[3] Lai C.D., Xie, M. and Murthy D.N.P. (2003) A Modified Weibull Distributions. IEEE Transactions on Reliability, 52, 33-37. https://doi.org/10.1109/TR.2002.805788

[4] Sarhan, A.M. and Zaindin, M. (2009) Modified Weibull Distribution. Applied Sciences, 11, 123-136.

[5] Famoye, F., Lee, C. and Olumolade, O. (2005) The Beta-Weibull Distribution. Journal of Statistical Theory and Applications, 4, 121-136.

[6] Silva, G.O., Ortega, E.M. and Cordeiro, G.M. (2010) The Beta Modified Weibull Distribution. Lifetime Data Analysis, 16, 409-430. https://doi.org/10.1007/s10985-010-9161-1

[7] Nadarajah, S., Cordeiro, G.M. and Ortega, E.M.M. (2011) General Results for the BetaModified Weibull Distribution. Journal of Statistical Computation and Simulation, 81, 1211-1232. https://doi.org/10.1080/00949651003796343

[8] Cordeiro, G.M., Ortega, E.M. and Nadarajah, S. (2010) The Kumaraswamy Weibull Distri- 
bution with Application to Failure Data. Journal of the Franklin Institute, 347, 1399-1429. https://doi.org/10.1016/j.jfranklin.2010.06.010

[9] Carrasco, M., Ortega, E.M. and Cordeiro, G.M. (2008) A Generalized Modified Weibull Distribution for Lifetime Modeling. Computational Statistics and Data Analysis, 53, 450462. https://doi.org/10.1016/j.csda.2008.08.023

[10] Sarhan, A.M. and Apaloo, J. (2013) Exponentiated Modified Weibull Extension Distribution. Reliability Engineering and System Safety, 112, 137-144. https://doi.org/10.1016/j.ress.2012.10.013

[11] Pham, H. and Lai, C.D. (2007) On Recent Generalizations of the Weibull Distribution. IEEE Transactions on Reliability, 56, 454-458. https://doi.org/10.1109/TR.2007.903352

[12] Murthy, D.N.P., Xie, M. and Jiang, R. (2003) Weibull Models. John Wiley and Sons, New York. https://doi.org/10.1002/047147326X

[13] Bebbington, M.S., Lai, C.D. and Zitikis, R. (2007) A Flexible Weibull Extension. Reliability Engineering \& System Safety, 92, 719-726. https://doi.org/10.1016/j.ress.2006.03.004

[14] AlKadim, K.A. and Boshi, M.A. (2013) Exponential Pareto Distribution. Mathematical Theory and Modeling, 3, 135-146.

[15] Bassiouny, A.H. Abdo, N.F. and Shahen, H.S. (2015) Exponential Lomax Distribution. International Journal of Computer Application, 13, 24-29.

[16] Kenney, J. and Keeping, E. (1962) Mathematics of Statistics. Volume 1, D. Van Nostrand Company, Princeton.

[17] Moors, J.J.A. (1998) A Quantile Alternative for Kurtosis. The Statistician, 37, 25-32. https://doi.org/10.2307/2348376

[18] Zwillinger, D. (2014) Table of Integrals, Series and Products. Elsevier, Amsterdam.

[19] Lawless, J.F. (2003) Statistical Models and Methods for Lifetime Data. John Wiley and Sons, New York, Vol. 20, 1108-1113.

[20] Akaike, H. (1974) A New Look at the Statistical Model Identification. IEEE Transactions on Automatic Control, 19, 716-723. https://doi.org/10.1109/TAC.1974.1100705

[21] Schwarz, G. (1978) Estimating the Dimension of a Model. Annals of Statistics, 6, 461-464. https://doi.org/10.1214/aos/1176344136

[22] Aarset, M.V. (1987) How to Identify Bathtub Hazard Rate. IEEE Transactions on Reliability, R-36, 106-108. https://doi.org/10.1109/TR.1987.5222310

\section{Submit or recommend next manuscript to SCIRP and we will provide best service} for you:

Accepting pre-submission inquiries through Email, Facebook, LinkedIn, Twitter, etc. A wide selection of journals (inclusive of 9 subjects, more than 200 journals)

Providing 24-hour high-quality service

User-friendly online submission system

Fair and swift peer-review system

Efficient typesetting and proofreading procedure

Display of the result of downloads and visits, as well as the number of cited articles

Maximum dissemination of your research work

Submit your manuscript at: http://papersubmission.scirp.org/

Or contact ojmsi@scirp.org 